\title{
Hamilton-Jacobi Formulation of KS Entropy for Classical and Quantum Dynamics
}

\author{
M. Hossein Partovi甘 \\ Department of Physics and Astronomy, California State University, Sacramento, California \\ 95819-6041
}

(November 3, 2018)

\begin{abstract}
A Hamilton-Jacobi formulation of the Lyapunov spectrum and KS entropy is developed. It is numerically efficient and reveals a close relation between the KS invariant and the classical action. This formulation is extended to the quantum domain using the Madelung-Bohm orbits associated with the Schroedinger equation. The resulting quantum KS invariant for a given orbit equals the mean decay rate of the probability density along the orbit, while its ensemble average measures the mean growth rate of configuration-space information for the quantum system.
\end{abstract}

05.45.Mt, 03.65.Ca, 03.67.-a, 05.45.Pq 
Chaos is a long-term instability exhibited by a dynamical system. It is quantitatively measured by the Lyapunov spectrum of characteristic exponents, which represents the principal rates of orbit divergence in phase space, or alternatively by the Kolmogorov-Sinai (KS) invariant, which quantifies the rate of information production by the dynamical system [1]. The KS invariant is a fundamental quantity that underlies the information dynamics of a broad class of dynamical systems well beyond classical mechanics. The results reported here show that it is intimately related to the classical action for Hamiltonian systems and to amplitude decay and configuration-space information for quantum systems. While chaos is a ubiquitous feature of nonlinear classical dynamics, it is conspicuously absent in finite quantum systems as these are known to be dynamically stable [2]. Nevertheless, the chaotic nature of a given classical Hamiltonian produces certain characteristic features in the dynamical behavior of its quantized version. These features have been studied extensively and are commonly referred to as "quantum chaos" [3, 四. They include short-term instabilities and diffusive behavior versus dynamical localization, characteristic spectral statistics, wave function scarring, and other effects. A quantitatively precise formulation of quantum dynamical instability smoothly straddling the quantum-classical transition, on the other hand, is at present lacking and is the subject of this work.

This Letter presents a general approach to the information dynamics of the quantumclassical transition based on the Hamilton-Jacobi (HJ) formalism. The formulation is exact and based on phase-space concepts, with the KS invariant playing a central role. The extension to the quantum domain is accomplished by means of the orbits introduced by Madelung [5] and later rediscovered by Bohm [6]. These quantum orbits are natural extensions of the classical phase-space flow to quantum mechanics, and provide the required bridge across the transition. The present approach yields several new results, including (i) a powerful and numerically efficient method for calculating the KS invariant for classical Hamiltonian systems, (ii) the striking finding that the quantum KS invariant for a given Madelung-Bohm (MB) orbit is equal to the mean decay rate of the probability density along the orbit, (iii) the result that the quantum KS invariant averaged over the ensemble of MB orbits equals the mean growth rate of configuration-space information, and (iv) a general and rigorous argument for the conjecture that the standard quantum-classical correspondence (or the classical limit) necessarily breaks down for classically chaotic Hamiltonians. Several other results, including those of numerical simulations for classical and quantum models, are presented and discussed.

Canonical formulation of chaos. Our objective here is a symplectically reduced formulation of the Lyapunov spectrum for a Hamiltonian system in terms of the classical action; see Ref. [7], hereafter referred to as "Paper I," for definitions and notation. Consider a classical system of $N$ degrees of freedom described by the canonical variables $\left\{q_{i}, p_{i}\right\}, i=$ $1, \ldots, N$, the Hamiltonian function $H(\mathbf{q}, \mathbf{p}, t)$, and Hamilton's principal function $S\left(\mathbf{q}, t, \mathbf{p}_{0}\right)$, with $\mathbf{p}_{0}$ denoting the initial momenta $[\mathbb{B}]$. Hamilton's equations can be compactly stated in matrix form as $\dot{\boldsymbol{\xi}}=\mathcal{J} \boldsymbol{\nabla}_{\xi} H(\boldsymbol{\xi}, t)$, where $\boldsymbol{\xi}$ stands for the $2 N$-dimensional phase-space vector $(\mathbf{q}, \mathbf{p})$. Here $\mathcal{J}$ is a real, antisymmetric matrix of order $2 N$ with a $2 \otimes N$ block form $\left(0_{N}, I_{N},-I_{N}, O_{N}\right)$, which is simply a listing of its blocks in the order $(11,12,21,22)$. The tangent dynamics of the system is described by the $2 N \times 2 N$, nonsingular matrix $\mathcal{T}_{\mu \nu}\left(t, \boldsymbol{\xi}_{0}\right) \stackrel{\text { def }}{=} \partial \xi_{\mu}\left(t, \boldsymbol{\xi}_{0}\right) / \partial \xi_{0 \nu}$, the sensitivity matrix, where $\boldsymbol{\xi}\left(t, \boldsymbol{\xi}_{0}\right)$ is the trajectory that starts from $\boldsymbol{\xi}_{0}$ at $t=0$. 
Our first task is to express $\mathcal{T}$ in terms of Hamilton's principal function $S$. Using the properties of $S$, we find the desired result in the block form

$$
\begin{aligned}
\mathcal{T}= & \left(S_{\mathbf{p}_{0} \mathbf{q}}{ }^{-1},-S_{\mathbf{p}_{0} \mathbf{q}}{ }^{-1} S_{\mathbf{p}_{0} \mathbf{p}_{0}},\right. \\
& \left.S_{\mathbf{q q}_{\mathbf{q}}} S_{\mathbf{p}_{0} \mathbf{q}}{ }^{-1}, \tilde{S}_{\mathbf{p}_{0} \mathbf{q}}-S_{\mathbf{q q}} S_{\mathbf{p}_{0} \mathbf{q}}{ }^{-1} S_{\mathbf{p}_{0} \mathbf{p}_{0}}\right),
\end{aligned}
$$

where $\left(S_{\mathbf{p}_{0} \mathbf{q}}\right)_{i j} \stackrel{\text { def }}{=} \partial^{2} S / \partial_{q_{j}} \partial_{p_{0 i}}[9]$. Note that $\mathcal{T}_{21} \mathcal{T}_{11}{ }^{-1}$ is a symmetric matrix (it equals $S_{\mathbf{q q}}$ ), a fact that is a direct consequence of the symplectic symmetry of the underlying Hamiltonian, which symmetry implies the condition $\mathcal{T} \mathcal{J} \tilde{\mathcal{T}}=\mathcal{J}[7]$. This allows us to transform the sensitivity matrix $\mathcal{T}$ to an upper-triangular block form $\Gamma$ according to $\Gamma \stackrel{\text { def }}{=} \Omega(\Theta) \mathcal{T}$, where $\Omega(\Theta) \stackrel{\text { def }}{=}(\cos \Theta,-\sin \Theta, \sin \Theta, \cos \Theta)$ and $\tan (\Theta) \stackrel{\text { def }}{=}-S_{\mathbf{q q}}$ [10]. Note that $\Theta$ is a real, symmetric matrix of order $N$, while $\Omega$ is orthogonal and symplectic. We shall refer to $\Theta$ as the symplectic phase matrix for reasons that will become clear in the following.

The upper-triangular form $\left(\Gamma_{11}, \Gamma_{12}, 0_{N}, \Gamma_{22}\right)$ of $\Gamma$, on the other hand, guarantees that $\Gamma_{11}^{-1}=\tilde{\Gamma}_{22}$, and that the upper (lower) half of the Lyapunov spectrum is obtained from the singular values of $\Gamma_{11}\left(\Gamma_{22}\right)$ as explained in Paper I. In particular, the KS entropy is given by 11

$$
k=\lim _{t \rightarrow \infty} \log \left[\operatorname{det}\left(\Gamma_{11}\right)\right] / t .
$$

The evolution equation for $\mathcal{T}$, Eq. (1) of Paper I, leads to the set of matrix equations

$$
\dot{\Gamma}_{11} \Gamma_{11}^{-1}=L^{A}+\mathcal{K}_{21}^{\Omega}, \quad \dot{\Omega}=\mathcal{L} \Omega, \quad L^{S}+\mathcal{K}_{11}^{\Omega}=0
$$

which can be used to calculate the Lyapunov spectrum efficiently. Here $\mathcal{L} \stackrel{\text { def }}{=}$ $\left(L^{A}, L^{S},-L^{S}, L^{A}\right), \mathcal{K}\left(t, \boldsymbol{\xi}_{0}\right) \stackrel{\text { def }}{=} \nabla_{\xi} \nabla_{\xi} H\left[\boldsymbol{\xi}\left(t, \boldsymbol{\xi}_{0}\right), t\right]$, and $\mathcal{K}^{\Omega} \stackrel{\text { def }}{=} \Omega \mathcal{K} \tilde{\Omega}$, where $L^{S}$ and $L^{A}$ are symmetric and antisymmetric matrices respectively. In particular, we note that the time average of the trace of the first equation in the set yields the KS invariant, i.e., $k=<\operatorname{tr}\left(\dot{\Gamma}_{11} \Gamma_{11}^{-1}\right)>$, where $<f>\stackrel{\text { def }}{=} \lim _{t \rightarrow \infty} t^{-1} \int_{0}^{t} f(\bar{t}) d \bar{t}$. Using the above set of equations, we find

$$
\begin{gathered}
\dot{\sigma}+\mathcal{K}_{11}+\mathcal{K}_{12} \sigma+\sigma \mathcal{K}_{21}+\sigma \mathcal{K}_{22} \sigma=0 \\
k=<\operatorname{tr}\left[\frac{\mathcal{K}_{11}-\mathcal{K}_{22}}{2} \sin 2 \Theta+\frac{\mathcal{K}_{12}+\mathcal{K}_{21}}{2} \cos 2 \Theta\right]>
\end{gathered}
$$

where $\sigma(t)$ is the restriction of $S_{\mathbf{q q}}=-\tan (\Theta)$ to a specific orbit and $\sigma(0)=0$. Equations (4) and (5), together with the equations of motion for the orbit, constitute a closed set from which the KS invariant can be calculated for any Hamiltonian system. The main computational burden is in solving the $N(N+1) / 2$ differential equations in Eq. (4). As with the results of Paper I, the above set of equations is free of exponentially growing quantities and does not require any orthonormality maintenance.

To illustrate the power of these equations, let us consider the standard form $H=\mathbf{p}^{2} / 2+$ $V(\mathbf{q}, t)$, where $\mathbf{q}$ and $\mathbf{p}$ are $N$-dimensional vectors. We then find $\mathcal{K}_{11}=\boldsymbol{\nabla}_{q} \boldsymbol{\nabla}_{q} V(\mathbf{q}, t)$, $\mathcal{K}_{12}=\mathcal{K}_{21}=0_{N}, \mathcal{K}_{22}=I_{N}$, and

$$
\dot{\sigma}+\sigma^{2}+\mathcal{K}_{11}=0
$$




$$
k=\frac{1}{2}<\operatorname{tr}\left(\mathcal{K}_{11}-1\right) \sin 2 \Theta>
$$

Moreover, using $\sigma=-\tan (\Theta)$ and Eq. (6), we can rewrite Eq. (7) in the remarkably simple form

$$
k=<\operatorname{tr} \sigma>_{p . v .}
$$

where "p.v." stipulates a principal-value evaluation [12]. Since $\operatorname{tr}(\sigma)$ equals $\nabla_{q}^{2} S$ along the orbit, Eq. (8) simply states that the KS invariant equals the time-average of the Laplacian of the action along the orbit.

We will consider two examples here. The first is defined by $N=3$ and $V(\mathbf{q})=$ $-\frac{1}{2}\left[\left(q_{1}-q_{2}\right)^{2}+\left(q_{2}-q_{3}\right)^{2}+\left(q_{3}-q_{1}\right)^{2}\right]+\left(q_{1}^{4}+2 q_{2}^{4}+3 q_{3}^{4}\right)$. Equations (6) and (17) together with Hamilton's equations, 13 in all, were integrated for a duration of $2.2 \times 10^{7}$ time units with the initial value of $H$ set at unity [13]. The result was $k=0.6126$, with the fluctuations never exceeding 0.0001 as the simulation was extended by one order of magnitude to the endpoint. In fact a $1 \%$ result was already achieved at about $10^{4}$ time units.

For a second example, we will derive explicit formulas for an $N$-dimensional kicked system (which is otherwise free) with $V(\mathbf{q}, t)=f(\mathbf{q}) \sum_{n=1}^{\infty} \delta(t-n T)$. Let $\left(\mathbf{q}_{n}, \mathbf{p}_{n}\right)$ be the coordinates just after the $n^{\text {th }}$ kick. Then $\left(\mathbf{q}_{n+1}, \mathbf{p}_{n+1}\right)=\left(\mathbf{q}_{n}, \mathbf{p}_{n}\right)+\left[\mathbf{p}_{n} T,-\nabla f\left(\mathbf{q}_{n+1}\right)\right]$. From Eq. (6), on the other hand, we find $\dot{\sigma}+\sigma^{2}=0$ for $t \neq n T$ and $\Delta \sigma_{n}=-\nabla \nabla f\left(\mathbf{q}_{n}\right)$ across the $n^{\text {th }}$ kick. The corresponding solution is easily found to be $\sigma(t)=\left(t-n T+\sigma_{n}^{-1}\right)^{-1}, n T<t<(n+1) T$. Thus the iteration map for $\sigma$ is

$$
\sigma_{n+1}=\left(\sigma_{n}^{-1}+T\right)^{-1}-\nabla \nabla f\left(\mathbf{q}_{n+1}\right),
$$

where $\sigma(0)=0$. We now use Eqs. (8) and (9) to find

$$
k=\lim _{N \rightarrow \infty}(N T)^{-1} \sum_{n=0}^{N-1} \ln \left|\operatorname{det}\left(1+\sigma_{n} T\right)\right|
$$

which, together with the iteration maps given above, provides an efficient algorithm for calculating the KS invariant for kicked systems. As an illustrative example, we chose $f(\mathbf{q})=$ $-\frac{1}{2}\left[\left(q_{1}-q_{2}\right)^{2}+\left(q_{2}-q_{3}\right)^{2}+\left(q_{3}-q_{1}\right)^{2}\right]+\left(q_{1}^{4}+q_{2}^{4}+q_{3}^{4}\right)$, with $T=1.0 \times 10^{-10}$. The value $k=1.5 \times 10^{5}$ was obtained in about $10^{7}$ iterations [14].

Note, incidentally, that in case an eigenvalue of $\sigma_{n}^{-1}$ is negative and less than $T$ in magnitude, $\sigma$ will encounter a simple pole in the $n+1^{\text {st }}$ cycle [other types of singularities are ruled out by Eq. (6)]. This behavior is generic for chaotic Hamiltonians (and common for all Hamiltonians), since otherwise a bounded $\sigma$ would, by virtue of Eq. (\$), lead to $k=0$. Correspondingly, the symplectic phase angles (eigenvalues of $\Theta$ ) go through $\left(m-\frac{1}{2}\right) \pi$ as $t$ goes through the $m^{\text {th }}$ singularity of that eigenvalue [we take $\Theta(0)=0$ ]. These poles occur when $\mathcal{T}_{11}$ becomes singular (as a matrix), and are the analogues of the so-called conjugate points which occur when $\mathcal{T}_{12}$ becomes singular [3]. Further insight is gained by considering these angles for quadratic potentials for which $\mathcal{K}_{11}$ is a constant, positive-definite matrix (and of course $k=0$ ). The solution to Eq. (6) is found to be $\sigma=-\mathcal{K}_{11}^{\frac{1}{2}} \tan \left(\mathcal{K}_{11}^{\frac{1}{2}} t\right)$. Thus the symplectic phases are given by $\theta_{i}=\arctan \left[\omega_{i} \tan \left(\omega_{i} t\right)\right]$, where $\left\{\omega_{i}\right\}$ are the characteristic frequencies of the system. Clearly, the $m$ th singularity associated with $\theta_{i}$ occurs for $\omega_{i} t=$ $\left(m-\frac{1}{2}\right) \pi$ as expected. The conjugate points occur midway between the latter at $\omega_{i} t=m \pi$. 
Extension to Quantum Mechanics. The MB formalism [5],6] associates a phase space flow with a quantum system by setting [15]

$$
\psi(\mathbf{x}, t) \stackrel{\text { def }}{=} \exp [i \mathrm{~S}(\mathbf{x}, t) / \hbar+\mathrm{R}(\mathbf{x}, t)]
$$

and treating S as Hamilton's principal function for an ensemble of orbits with configurationspace density $\exp (2 \mathrm{R})$. These orbits are defined according to

$$
\dot{\mathrm{q}}\left(t, \mathrm{q}_{0}, \mathrm{p}_{0}\right)=\mathrm{p}\left(t, \mathrm{q}_{0}, \mathrm{p}_{0}\right)=\nabla \mathrm{S}\left[\mathrm{q}\left(t, \mathrm{q}_{0}, \mathrm{p}_{0}\right), t\right],
$$

where $\mathrm{q}_{0}=\mathrm{q}\left(0, \mathrm{q}_{0}, \mathrm{p}_{0}\right)$. Here $\psi(\mathbf{x}, t)$ is a solution of the Schroedinger equation with the Hamiltonian $\mathbf{p}^{2} / 2+V(\mathbf{x}, t)$. It is readily verified that the expectation value of any observable in the state $\psi(\mathbf{x}, t)$ is given by its average over the ensemble of orbits defined above. In particular, the ensemble average of Eq. (12) will lead to Ehrenfest's equations.

The correspondence thus established allows us to define the quantum KS invariant for a given orbit as

$$
\mathrm{k} \stackrel{\text { def }}{=}<\nabla^{2} \mathrm{~S}>_{p . v .}
$$

in strict analogy to Eq. (8). It is worth emphasizing here that the averaging process in Eq. (13) is with respect to the time along the MB orbit to which $\mathrm{S}$ is restricted.

Intuitively, one would expect that orbits neighboring a hypothetical chaotic orbit in the ensemble diverge from it on the average, thus causing the orbit density along the chaotic orbit to decrease with a mean rate related to k. Remarkably, this expectation is fully realized by the Schroedinger equation. To see this, consider the equation of motion for $\mathrm{R}(\mathbf{x}, t)$ as inherited from the Schroedinger equation: $\partial \mathrm{R} / \partial t+\nabla \mathrm{R} \cdot \nabla \mathrm{S}=-\frac{1}{2} \nabla^{2} \mathrm{~S}$. An inspection of this equation shows that its characteristic curves are the MB orbits, so that it takes the following form along those orbits:

$$
d \mathrm{R}\left(\mathrm{q}\left(t, \mathrm{q}_{0}, \mathrm{p}_{0}\right), t\right) / d t=-\frac{1}{2} \nabla^{2} \mathrm{~S}\left[\mathrm{q}\left(t, \mathrm{q}_{0}, \mathrm{p}_{0}\right), t\right] .
$$

Using this in Eq. (13), we find $\mathrm{k}=-2<d \mathrm{R} / d t>_{p . v \text {. }}$, or equivalently

$$
\mathbf{k}=-<d \ln \left|\psi\left[\mathbf{q}\left(t, \mathbf{q}_{0}, \mathbf{p}_{0}\right), t\right]\right|^{2} / d t>_{p . v .} .
$$

This exact result states that the quantum KS invariant for a given orbit is the mean decay rate of the probability density along the orbit. We mention in passing that if $\psi$ in Eq. (15) is replaced with its classical limit $\psi_{\text {s.c. }}=\left[\operatorname{det}\left(S_{\mathbf{p}_{0} \mathbf{q}}\right)\right]^{\frac{1}{2}} \exp [i S(\mathbf{x}, t) / \hbar]$, the result is the classical $\mathrm{KS}$ invariant $k$ [9].

Let us consider the implications of Eq. (15) for classically chaotic systems, for which $k \neq 0$ while $\mathrm{k}=0$. For a chaotic orbit in classical dynamics, there is no integral of motion in phase-space except possibly energy. For the ensemble of MB orbits, on the other hand, the defining equations (12) provide $N$ such integrals (upon eliminating $\dot{\mathrm{q}}$ ) if $\nabla \mathrm{S}$ is a longterm predictable, or computable, function. But $\nabla \mathrm{S}$ is computable, since the Schroedinger equation for finite-dimensional, bounded systems is known to be free of sensitivity to initial conditions [2]. The inevitable conclusion is that while $\nabla S$ (classical) is not computable for chaotic systems, $\nabla \mathrm{S}$ (quantum mechanical) is, regardless of how small $\hbar$ may be. Clearly, 
the statement that $\nabla S$ reduces to $\nabla S$ as $\hbar \rightarrow 0$, often referred to as the classical limit, cannot hold for chaotic Hamiltonians. Recalling that regular Hamiltonians are very special while chaotic ones are by far the more typical class, we recognize the very limited validity of the classical limit.

The above considerations confirm the view that the absence of sensitivity to initial conditions in the quantum dynamics of classically chaotic Hamiltonians originates in the breakdown of the classical limit itself for large times. Stated otherwise, the two limiting procedures $\hbar \rightarrow 0$ and $t \rightarrow \infty$ do not commute for classically chaotic Hamiltonians [2, 1, 16]. How is this related to the issue of computability raised above? Long-time unpredictability is precisely the condition that characterizes $\nabla S$ as uncomputable, in contrast to $\nabla S$ which is computable by way of the Schroedinger equation. Of course, for sufficiently small times the two can be arbitrarily close. That a quantum system can temporarily exhibit dynamic instability was recognized early in a numerical study of the quantum kicked rotor model [17], and has since been investigated extensively [四].

Indeed it is useful to restate the general arguments above in more explicit terms for this widely studied model. Classically, the kicked rotor is a driven, one-dimensional system with chaotic orbits for appropriate values of the driving force. The MB orbits for this system, on the other hand, are governed by (12), which amounts to a single, non-autonomous first-order differential equation. As such, this system cannot possibly be chaotic [18, implying that in the course of time the quantum orbits will deviate arbitrarily far from the classical ones. Therefore the classical limit must of necessity fail for this system. Correspondingly, $\mathrm{k}$ as measured according to Eq. (13) must vanish.

In a numerical simulation of the quantum kicked rotor for the purpose of verifying this prediction, we integrated Eq. (12) together with the Schroedinger equation, and measured k along the resulting MB orbit using Eq. (13]). The result was zero [19], thus verifying the predicted regularity of the orbit. An alternative calculation using a pair of nearby orbits reconfirmed the regularity of these orbits. This raises the question of whether $\mathrm{k}$ can ever be positive for this model. To answer this question, we explored the behavior of the hybrid quantity $k^{h y b} \stackrel{\text { def }}{=}<\nabla^{2} \mathbf{S}\left[\mathbf{q}\left(t, \mathbf{q}_{0}, \mathbf{p}_{0}\right), t\right]>_{p . v .}$. Observe that here we are considering the quantum objects $\psi$ and $\mathbf{S}$ as evaluated along the classical orbit $\mathbf{q}\left(t, \mathbf{q}_{0}, \mathbf{p}_{0}\right.$ ) (in place of $\mathrm{MB}$ orbits). Note that while $k^{h y b}$ is not required to vanish by the general arguments above, there is no a priori reason to the contrary either, despite the chaotic nature of the classical orbit. Numerical results, however, showed nonzero values of $k^{h y b}$ for a variety of conditions, in striking contrast to the behavior of $\mathrm{k}$. This comparison clearly identifies the difference between the chaotic classical orbits and their quantum counterparts as the origin of the dynamical stability of the quantum kicked rotor. It also indicates that the mean decay rate of $\psi\left[\mathbf{q}\left(t, \mathbf{q}_{0}, \mathbf{p}_{0}\right), t\right]$, the restriction of the wavefunction to the classical orbit, is a discriminating footprint of classical chaos in quantum dynamics. A systematic study of this intriguing phenomenon must await a better analytical understanding of the hybrid object $\psi\left[\mathbf{q}\left(t, \mathbf{q}_{0}, \mathbf{p}_{0}\right), t\right]$.

Thus far we have considered $k$ for single MB orbits. What is the MB ensemble average of $k$, denoted by $\bar{k}$ ? Recalling that the MB ensemble average is the same as the quantum mechanical expectation value, we find from Eq. (15) or (13)

$$
\overline{\mathrm{k}}=\lim _{t \rightarrow \infty} \frac{1}{t} \int d \mathbf{q}|\psi(\mathbf{q}, t)|^{2} \ln \left[|\psi(\mathbf{q}, t)|^{-2}\right] .
$$


The integral occurring in (16) is recognized as the information associated with the probability density $|\psi(\mathbf{q}, t)|^{2}$. It is actually the entropy associated with an idealized position measurement, as shown in Ref. [20]. Thus we have arrived at the result that the ensemble average of the quantum KS invariant equals the mean growth rate of the position measurement entropy. Precisely this result was proposed in Ref. [21] on the basis of more intuitive considerations. The fact that the original information-theoretical significance of the KS entropy has naturally re-emerged in the quantum domain may be viewed as an affirmation of the present approach.

As is well known [17,4], short-term measurements of $k$ typically show positive values. In light of Eqs. (15) and (16), this suggests a tendency of the probability density to decrease along certain $\mathrm{MB}$ orbits thereby developing information, i.e., structure and contrast, in configuration space. Presumably, points of low concentration would represent the interiors of regions of instability corresponding to classical chaos, with the boundaries as points of high concentration. This scenario is reminiscent of the scarring phenomena discovered by Heller [22] and merits further exploration.

This work was supported in part by a research grant from California State University, Sacramento. 


\section{REFERENCES}

* Electronic address: hpartovi@csus.edu

[1] J. P. Eckmann and D. Ruelle, Rev. Mod. Phys. 57, 617 (1985).

[2] M. H. Partovi, Phys. Rev. A 45, R555 (1992).

[3] M. C. Gutzwiller, Chaos in Classical and Quantum Mechanics (Springer-Verlag, New York, 1990).

[4] Quantum Chaos, edited by G. Casati and B. Chirikov (Cambridge University Press, Cambridge, 1995).

[5] E. Madelung, Z. F. Physik 40, 332 (1926).

[6] D. Bohm, Phys. Rev. 84, 166 (1951).

[7] M. H. Partovi, Phys. Rev. Lett. 82, 3424 (1999).

[8] H. Goldstein, Classical Mechanics, 2nd ed. (Addison-Wesley, Reading, 1980).

[9] Note that $\operatorname{det}\left(S_{\mathbf{p}_{0} \mathbf{q}}\right)$ is the van Vleck determinant in our formulation; J. van Vleck, Proc. Nat. Acad. Sci. 14, 178 (1928).

[10] An entirely equivalent formulation results if we consider Hamilton's principal function as $S\left(\mathbf{q}, t, \mathbf{q}_{0}\right)$ and $\Gamma$ as a lower-triangular matrix.

[11] While in general $k$ is associated with some phase-space region and defined independently of the Lyapunov spectrum, we are relying on Pesin's result and the ergodicity of the chaotic orbit in writing Eq. (2); see Paper I.

[12] In deriving Eq. ( 8), we have dropped the quantity $\operatorname{tr}<d \ln \left(1+\sigma^{2}\right) / d t>$ which vanishes if $\sigma$ is bounded everywhere. However, Eq. (6) implies the simple pole behavior $\sigma \sim$ $\left(t-t_{s}\right)^{-1}$ near any singularity $t=t_{s}$. Since the principal-value contribution to Eq. (7) from a simple pole vanishes, the result follows.

[13] The simulation was performed on an HP 9000 computer using the Bulirsch-Stoer method for a duration of $2.2 \times 10^{7}$ units in $1.5 \times 10^{8}$ steps, with the local accuracy set at $10^{-10}$. The starting point was $\boldsymbol{\xi}_{0}=\left(-0.075,0.1,-0.2,-0.25,0.12, \xi_{8}\right)$, where $\xi_{8} \geq 0$ was determined from the energy condition. Stoermer's scheme was used for Hamilton's equations, resulting in an energy drift of less than $1.8 \times 10^{-8}$ by the end of the simulation.

[14] The starting point for this simulation was $\boldsymbol{\xi}_{0}=(1,0,0,0,0,0)$. The fluctuations remained at the $1-2 \%$ level as the simulation was extended to $10^{8}$ iterations.

[15] Note the non-standard definition of $\mathrm{R}$ here.

[16] J. Robinson and M. Berry, J. Phys. A 25, L961 (1992).

[17] G. Casati, B. Chirikov, F. Izrailev, and J. Ford, in Stochastic Behavior in Classical and Quantum Systems, Lecture Notes in Physics 93, edited by G. Casati and J. Ford (Springer, Berlin, 1979).

[18] Chaos is not possible with less than three autonomous, or two non-autonomous, firstorder differential equations.

[19] Specifically, the measured value of $k$ decreased, on the average, in inverse proportion to the length of the simulation.

[20] M. H. Partovi, Phys. Rev. Lett. 50, 1883 (1983).

[21] M. H. Partovi, Phys. Lett. A 151, 389 (1990).

[22] E. J. Heller, Phys. Rev. Lett. 53, 1515(1984). 